\title{
On the enumeration of $d$-minimal permutations
}

\author{
Mathilde BouveL* \\ LaBRI UMR 5800, Université de Bordeaux and CNRS, \\ 351, cours de la Libération, 33405 Talence cedex, France \\ bouvelolabri.fr \\ Luca FERRARI \\ Dipartimento di Sistemi e Informatica, Università degli Studi di Firenze, \\ Viale Morgagni 65, 50134 Firenze, Italy \\ ferrari@dsi.unifi.it
}

\begin{abstract}
We suggest an approach for the enumeration of minimal permutations having $d$ descents which uses skew Young tableaux. We succeed in finding a general expression for the number of such permutations in terms of (several) sums of determinants. We then generalize the class of skew Young tableaux under consideration; this allows in particular to discover some presumably new results concerning Eulerian numbers.
\end{abstract}

\section{Introduction}

This article deals with minimal permutations with $d$ descents (also called $d$-minimal permutations here). This family of permutations has been introduced in BoRo in the study of the whole genome duplication-random loss model of genome rearrangement. In this context, genomes are represented by permutations, and minimal permutations with $d=2^{p}$ descents are the basis of excluded patterns that describes the class of permutations that can be obtained from the identity with cost at most $p$.

In order to describe properties of this class of permutations, its basis has been studied, and the first natural question to address is to count how many excluded patterns it contains. In $\mathrm{BP}$ some partial results on the enumeration of minimal permutations with $d$ descents have been obtained: namely, minimal permutations with $d$ descents and of size $n$ have been enumerated by closed formulas, for $n=d+1, d+2$ and $2 d(d+1$ and $2 d$ being lower and upper bounds for the size of a minimal permutation with $d$ descents - see

\footnotetext{
* Mathilde Bouvel conveys special acknowledgements to the Dipartimento di Sistemi e Informatica for the kind hospitality during her visit in May 2008 in which this research started.
} 
[BP]). In [MY], further results on the enumeration of minimal permutations with $d$ descents have been obtained using multivariate generating functions, allowing in particular to derive a closed formula enumerating those of size $2 d-1$ as well as some asymptotic results.

In this work we offer an alternative approach for the enumeration of minimal permutations with $d$ descents, making extensive use of a bijection between these permutations and a family of skew Young tableaux. This gives a general formula for the number $p_{d+k, d}$ of minimal permutations with $d$ descents and of size $d+k$, as a sum of determinants of matrices (Theorem 4.2). This expression for $k=d$ will specialize into a determinant expression of Catalan numbers which is believed to be new. When specializing it for $k=3$, it also allows us to give a closed formula for $p_{d+3, d}$ (Theorem 4.4). Finally, the family of skew Young tableaux under consideration has a natural generalization which is investigated in Section 5 .

\section{Preliminary definitions and results}

For any integer $n, S_{n}$ denotes the set of permutations of [1..n]. A permutation $\sigma \in S_{n}$ will be represented either by the word $\sigma(1) \ldots \sigma(n)$ or by the $n \times n$ grid, where a cell contains a dot if and only if it is at coordinates $(i, \sigma(i))$ for some $i \in[1 . . n]$.

The pattern involvement order on permutations $[\mathrm{P}]$ is defined as follows. A permutation $\pi \in S_{k}$ is involved in (or is a pattern of) $\sigma \in S_{n}$ when there exist integers $1 \leq i_{1}<\ldots<i_{k} \leq n$ such that $\pi$ and $\sigma\left(i_{1}\right) \ldots \sigma\left(i_{k}\right)$ are orderisomorphic sequences, i.e. they are such that $\pi(\ell)<\pi(m) \Leftrightarrow \sigma\left(i_{\ell}\right)<\sigma\left(i_{m}\right)$ for all $\ell, m \in[1 . . k]$.

A descent in a permutation $\sigma \in S_{n}$ is an integer $i \in[1 . .(n-1)]$ such that $\sigma(i)>\sigma(i+1)$. Similarly, an ascent is an integer $i \in[1 . .(n-1)]$ such that $\sigma(i)<\sigma(i+1)$.

A minimal permutation with $d$ descents, or d-minimal permutation, of length $n$ is a permutation of $S_{n}$ that is minimal in the sense of the patterninvolvement relation for the property of having $d$ descents. In other words, it is a permutation with $d$ descents such that, when removing any of its entries and suitably renaming the remaining elements, the resulting permutation of $S_{n-1}$ has $d-1$ descents. For instance (see Figure 11), the permutation $\sigma=141293135151062111874$ is minimal with 11 descents, as it has exactly 11 descents and every permutation it involves as a pattern has at most 10 descents.

In [BP], minimal permutations with $d$ descents have been characterized as follows:

Theorem 2.1 A permutation $\sigma$ is minimal with $d$ descents if and only if it has exactly $d$ descents and its ascents i satisfy the "diamond property", 


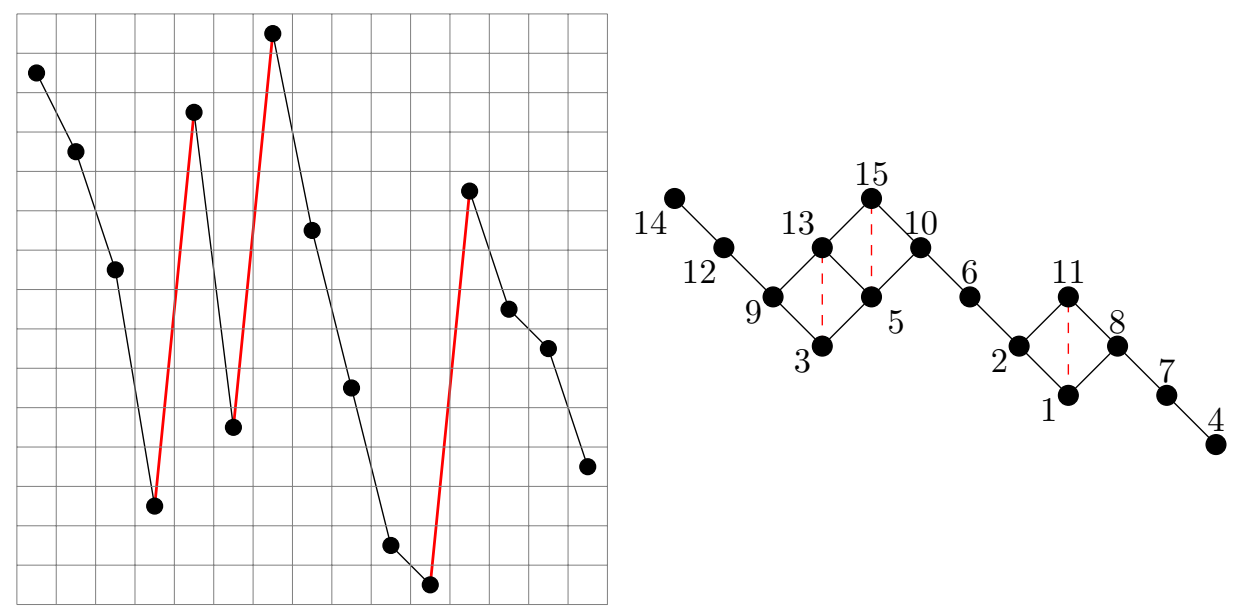

Figure 1: The 11-minimal permutation $\sigma=141293135151062111874$, and the corresponding poset.

i.e. are such that $2 \leq i \leq n-2$ and $\sigma(i-1) \sigma(i) \sigma(i+1) \sigma(i+2)$ forms an occurrence of either the pattern 2143 or the pattern 3142.

As explained in $[\mathrm{BP}]$, this characterization allows to represent $d$-minimal permutations by means of certain labelled posets. These posets, labelled with the integers from 1 to $n$, are made of chains somehow linked by diamondshaped structures (corresponding to the ascents of the permutation). Figure 11 shows an example of this one-to-one correspondence. Notice that each of these labelled posets represents a unique $d$-minimal permutation, whereas the underlying unlabelled poset can be seen as representing a set of $d$ minimal permutations (those that are in correspondence with a legal labelling of the poset).

Posets and labelled posets in these families are in one-to-one correspondence with skew Ferrers diagrams and skew Young tableaux having special properties. These combinatorial objects have been widely studied in the literature, in particular from an enumerative point of view (see, for instance, the recent paper [BaRd]). In the following sections, we explicitly describe the correspondence between unlabelled (resp. labelled) posets and skew Ferrers diagrams (resp. skew Young tableaux), as well as some enumerative results on these objects, and how they can be used for our purposes.

\section{Connection with skew Young tableaux}

In order to explain how the poset representation of $d$-minimal permutations described in the previous section can be conveniently interpreted by using skew Young tableaux, we first need to recall some definitions. 
An integer partition of an integer $n$ is a sequence of positive integers $\lambda=\left(\lambda_{1}, \ldots, \lambda_{k}\right)$ such that $\lambda_{i} \geq \lambda_{i+1}$ for $1 \leq i \leq k-1$ and $\sum_{i=1}^{k} \lambda_{i}=n$. The integer $n$ is called the size of the integer partition, and we write $n=|\lambda|$. The number of parts $k$ will be denoted by $k=\ell(\lambda)$ (this is also called the length of $\lambda$ ).

An integer partition $\lambda=\left(\lambda_{1}, \ldots, \lambda_{k}\right)$ can be represented by its Ferrers diagram, which is obtained by drawing $k$ rows of contiguous unit cells, from top to bottom, such that row $i$ contains $\lambda_{i}$ cells, and with the first cells of these $k$ rows vertically aligned. An example is shown in Figure 2. We will also denote by $\lambda$ the Ferrers diagram associated with the integer partition $\lambda$. The size $|\lambda|$ obviously corresponds to the number of cells of the Ferrers diagram, and the number of rows is given by $\ell(\lambda)$.

For our purposes, a Young tableau is a filling of a Ferrers diagram $\lambda$ using distinct positive integers from 1 to $n=|\lambda|$, with the properties that the values are (strictly) decreasing along each row and each column of the Ferrers shape. This constitutes a slight departure from the classical definition, which requires the word "increasing" instead of the word "decreasing". However, it is clear that all the properties and results on (classical) Young tableaux can be translated into our setting by simply replacing the total order " $\leq$ " with the total order " $\geq$ " on $\mathbf{N}$. In Figure 2 a Young tableau of shape $\lambda=$ $(8,6,3,3,2,1)$ is shown. Like for Ferrers diagrams, the size of a Young tableau is given by the number of its cells.

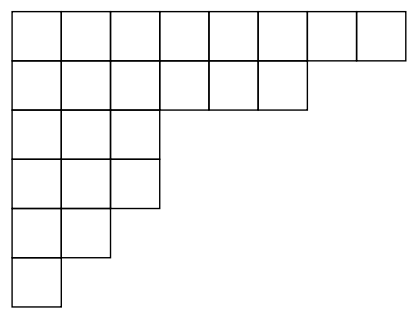

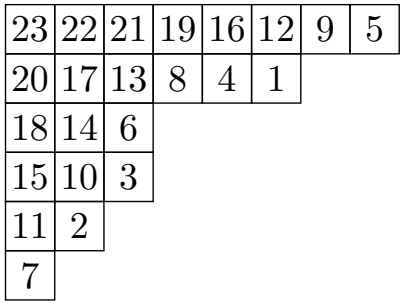

Figure 2: The Ferrers diagram associated with the integer partition $\lambda=$ $(8,6,3,3,2,1)$, and a Young tableau on this shape.

The main definition we need in our work is that of a skew Young tableau. The definition can be given exactly as for a Young tableau, with the only difference that the underlying shape consists of a Ferrers diagram $\lambda$ with a Ferrers diagram $\mu$ removed (starting from the top-left corner). Such a skew shape is usually denoted $\lambda \backslash \mu$. We refer the reader to [St] for the formal definition and some important facts concerning the enumeration of skew Young tableaux. In Figure 3 a skew Young tableau of skew shape $(8,6,3,3,2,1) \backslash(3,2,2,1)$ is depicted. As before, the size of a skew Young tableau denotes its number of cells. 

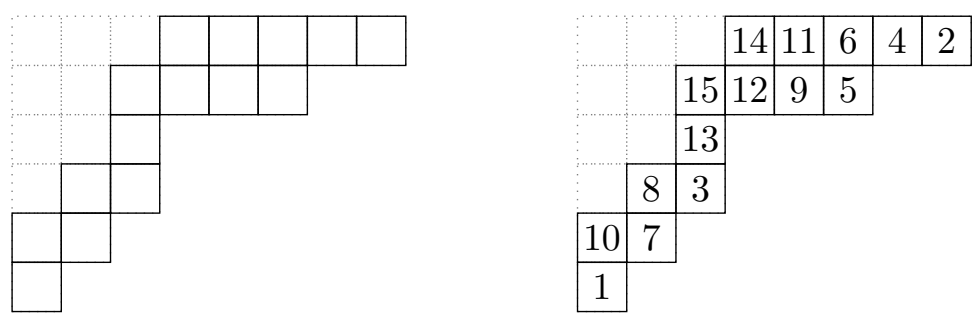

Figure 3: The skew shape $(8,6,3,3,2,1) \backslash(3,2,2,1)$, and a skew Young tableau on this shape.

As announced at the beginning of the present section, we can translate the poset representation of a $d$-minimal permutation into a suitable skew Young tableaux.

Proposition 3.1 The set of d-minimal permutations of length $d+k$ is in bijection with the set of skew Young tableaux whose skew shapes $\lambda \backslash \mu$ satisfy $|\lambda \backslash \mu|=d+k$, having $k$ rows and such that two consecutive rows have precisely two columns in common.

Proof. A $d$-minimal permutation of length $d+k$ consists of $k$ descending runs and, denoting with $a, b, c, d$ four consecutive elements such that $a, b$ and $c, d$ belong to different descending runs, then necessarily $a>b, c>d, a<c$ and $b<d$ (see Theorem 2.1). Then, starting from a $d$-minimal permutation $\pi$ of length $d+k$, one can construct a skew Young tableau as follows: starting from the bottom, the $i$-th row of the tableau consists of the elements of the $i$-th descending run of $\pi$; moreover two consecutive rows are required to have exactly two columns in common. The resulting tableau is skew Young thanks to the above recalled diamond property of $d$-minimal permutations.

In Figure 1 the skew Young tableau determined by the permutation whose poset representation is given in Figure 1 is shown.

Example. For $k=2$ and $d=4$, the set of 4-minimal permutations of length 6 is in bijection with the set consisting of all skew Young tableaux of one of the following skew shapes:

- $(4,2) \backslash \emptyset$, i.e. $\square$,

- $(4,3) \backslash(1)$, i.e. $\square \square$,

- $(4,4) \backslash(2)$, i.e. 

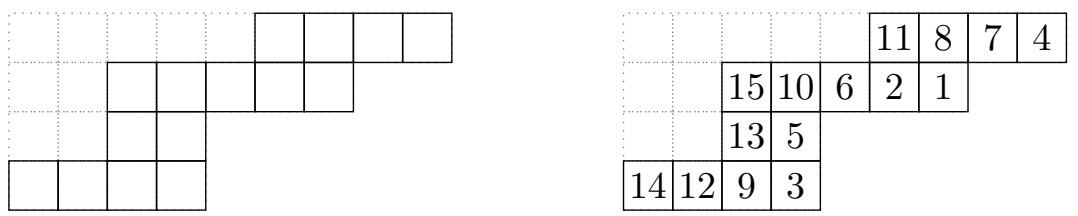

Figure 4: The skew shape corresponding to the underlying unlabelled poset of Figure 1, and the skew Young tableau corresponding to the permutation $\sigma$ of Figure 1 .

There are respectively 9, 14 and 9 skew Young tableaux of these shapes, giving a total of 324 -minimal permutations of length 6 .

Remarks. Let $\pi$ be a $d$-minimal permutation and suppose it has $k$ descending runs. Obviously this means that $\pi$ has $k-1$ ascents and that $|\pi|=d+k$. Moreover, suppose that $\lambda \backslash \mu$ is the skew shape associated with $\pi$. Recall that $\ell(\lambda)$ and $\ell(\mu)$ denote the number of rows of $\lambda$ and $\mu$ respectively. Finally, let $\ell_{i}$ be the length of the $i$-th descending run of $\pi$. Some straightforward consequences of the above bijection are the following:

1. The skew shape associated with $\pi$ is connected.

2. $\ell(\lambda)=k$.

3. Set $\lambda=\left(\lambda_{1}, \lambda_{2}, \ldots \lambda_{k}\right)$. Then $\lambda_{i}=\sum_{j=1}^{k-i+1} \ell_{j}-2(k-i)$.

4. $\ell(\mu)<\ell(\lambda)$ and, more precisely, $\ell(\mu)=(\ell(\lambda)-1)-$ \#(starting descending runs of length 2 in $\pi$ ).

5. Set $\mu=\left(\mu_{1}, \mu_{2}, \ldots \mu_{k}\right)$. Then $\mu_{i}=\lambda_{i+1}-2$.

\section{Some enumerative results}

The main goal of the present section is to enumerate $d$-minimal permutations of length $n$, with $d+1 \leq n \leq 2 d$. A general result in this direction can be obtained by considering the above described bijection with skew Young tableaux. In particular, an interesting result due to Aitken is our starting point.

Theorem 4.1 (迹) Let $\lambda \backslash \mu$ be a skew shape, with $|\lambda \backslash \mu|=N$ and $\ell(\lambda)=n$. Then, the number $f^{\lambda \backslash \mu}$ of skew Young tableaux of shape $\lambda \backslash \mu$ is

$$
f^{\lambda \backslash \mu}=N ! \operatorname{det}\left(\frac{1}{\left(\lambda_{i}-\mu_{j}-i+j\right) !}\right)_{i, j=1 . . n} .
$$


Formula (这) can be deduced from the well known Jacobi-Trudi identity, as shown, for instance, in [St2]. Moreover, in such a formula the entry $(i, j)$ of the considered matrix is intended to be 0 if the expression $\lambda_{i}-\mu_{j}-i+j$ is negative.

The main result of this section is essentially a corollary of Theorem 4.1, in the case in which the skew shape $\lambda \backslash \mu$ has the properties of Proposition 3.1 .

Theorem 4.2 Denote by $p_{d+k, d}$ the number of d-minimal permutations of length $d+k$ (so that $1 \leq k \leq d)$. Then

$$
p_{d+k, d}=\sum_{\substack{a_{1}, a_{2}, \ldots, a_{k} \geq 2 \\ a_{1}+a_{2}+\cdots+a_{k}=d+k}}(d+k) ! \cdot \operatorname{det}\left(A\left(a_{1}, \ldots, a_{k}\right)\right),
$$

where $A\left(a_{1}, \ldots, a_{k}\right)$ is the following matrix:

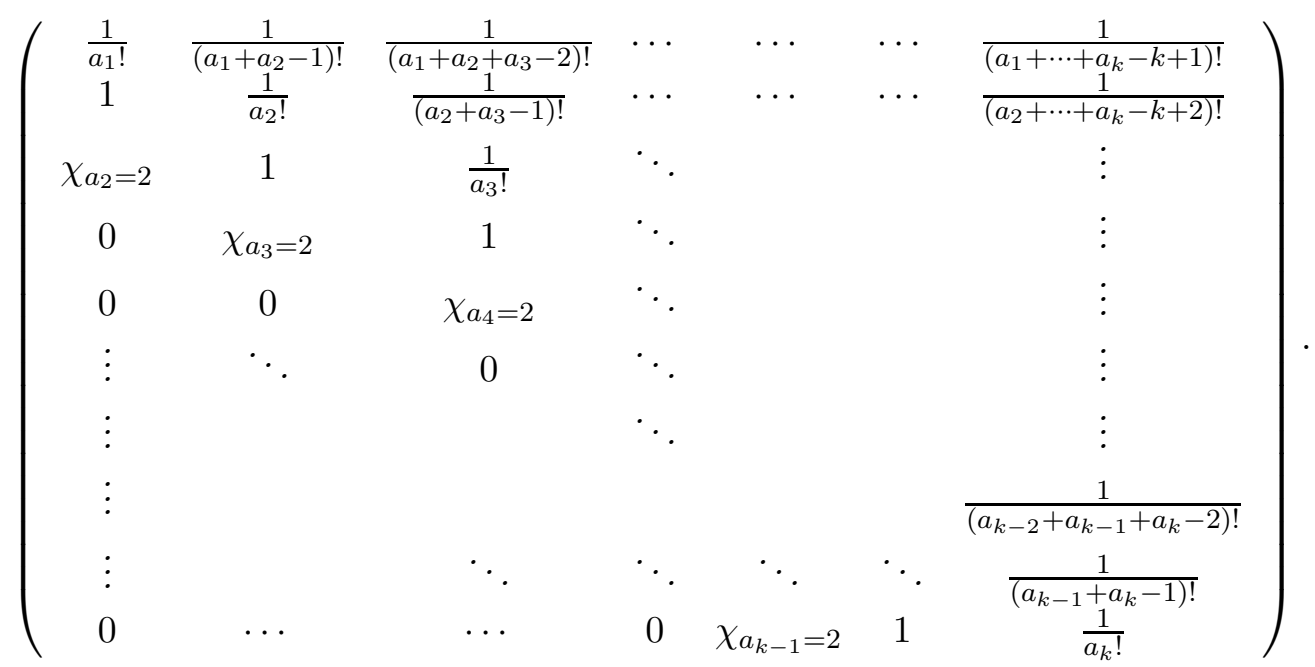

Here $\chi_{P}$ denotes the characteristic function of the property $P$ (i.e., $\chi_{P}=1$ when $P$ is true and $\chi_{P}=0$ otherwise). In other words, $A\left(a_{1}, \ldots, a_{k}\right)$ is the $k \times k$ matrix whose entries $a_{i, j}$ obey the following equalities:

$$
\begin{aligned}
a_{i, j} & =\frac{1}{\left(a_{i}+\cdots+a_{j}+i-j\right) !}, \quad \text { when } i \leq j, \\
a_{i, i-1} & =1, \\
a_{i, i-2} & =\chi_{a_{i-1}=2}, \\
a_{i, j} & =0, \quad \text { when } i>j+2 .
\end{aligned}
$$

Proof. Theorem 4.1 ensures that

$$
p_{d+k, d}=\sum_{\lambda \backslash \mu}(d+k) ! \operatorname{det}\left(\frac{1}{\left(\lambda_{i}-\mu_{j}-i+j\right) !}\right)
$$


where the sum is over all skew shapes $\lambda \backslash \mu$ of size $d+k$ having $k$ rows and such that two consecutive rows have exactly two columns in common.

For such a skew shape $\lambda \backslash \mu$, let us define the sequence $a=\left(a_{1}, a_{2}, \ldots, a_{k}\right)$ by $a_{i}=\lambda_{i}-\mu_{i}, 1 \leq i \leq k$. The sequence $a$ is such that $a_{1}, a_{2}, \ldots, a_{k} \geq 2$ and $a_{1}+a_{2}+\cdots+a_{k}=d+k$. From the remark at the end of the previous section, we additionally have that $\mu_{k}=0$ (point 4.) and that $\mu_{i}=\lambda_{i+1}-2$ for all $1 \leq i \leq k-1$ (point 5.). It is now trivial matter to check that the sequence $a=\left(a_{1}, a_{2}, \ldots, a_{k}\right)$ completely and uniquely determines $\lambda \backslash \mu$. Hence, the sum in the above formula can be taken over sequences $a=\left(a_{1}, a_{2}, \ldots, a_{k}\right)$ such that $a_{1}, a_{2}, \ldots, a_{k} \geq 2$ and $a_{1}+a_{2}+\cdots+a_{k}=d+k$.

In what follows, we give expressions of the entries $a_{i, j}=\frac{1}{\left(\lambda_{i}-\mu_{j}-i+j\right) !}$ in terms of $\left(a_{1}, a_{2}, \ldots, a_{k}\right)$.

If $i \leq j$, then, by Theorem 4.1, $a_{i, j}=\frac{1}{\left(\lambda_{i}-\mu_{j}-i+j\right) !}$. Thanks to the remarks stated at the end of the previous section, we have that:

$a_{i}+a_{i+1}+\ldots+a_{j}=\left(\lambda_{i}-\mu_{i}\right)+\left(\lambda_{i+1}-\mu_{i+1}\right)+\ldots\left(\lambda_{j}-\mu_{j}\right)=\lambda_{i}-\mu_{j}+2(j-i)$.

This yields for the denominator of the above fraction the following expression (leaving aside the factorial):

$$
\lambda_{i}-\mu_{j}-i+j=a_{i}+a_{i+1}+\ldots+a_{j}+i-j,
$$

as desired.

If $j=i-1$, then we have immediately:

$$
a_{i, i-1}=\frac{1}{\left(\lambda_{i}-\mu_{i-1}-1\right) !}=\frac{1}{(2-1) !}=1 .
$$

Concerning the case $j=i-2$, since $\mu_{i-1}=\lambda_{i}-2$ and $\mu_{i-2} \geq \mu_{i-1}$, we observe that $\lambda_{i}-\mu_{i-2} \leq 2$, and that the equality holds precisely when $\mu_{i-1}=\mu_{i-2}$, i.e. when $a_{i}=\lambda_{i-1}-\mu_{i-1}=2$. Thus we get:

$$
a_{i, i-2}=\frac{1}{\left(\lambda_{i}-\mu_{i-2}-i+i-2\right) !}=\chi_{a_{i-1}=2} .
$$

Finally, if $i>j+2$, then the denominator of $a_{i, j}$ is easily seen to be negative, hence $a_{i, j}=0$.

From a theoretical point of view, Theorem 4.2 completely solves the problem of the enumeration of $d$-minimal permutations with respect to their length, giving a formula for $p_{d+k, d}$. Unfortunately, it is clear that such a formula is very difficult to use in concrete cases, due to its intrinsic complexity. However, using our result we are able to rediscover some known cases (namely $k=1,2$ ) and to give an interpretation of Catalan numbers (corresponding to the case $k=d$ ), as well as to get a formula for the case $k=3$ (that is, $d$-minimal permutations of length $d+3$ ), which was first 
discovered in [MY] with different methods in terms of generating functions. The formula we derive has been found with the help of Maple.

We start by collecting in a single theorem the known cases $d=1,2$, showing how they can be derived from Theorem 4.2 .

Theorem $4.3([\overrightarrow{B P})$ The following equalities hold:

$$
\begin{aligned}
& p_{d+1, d}=1 \\
& p_{d+2, d}=2^{d+2}-(d+1)(d+2)-2 .
\end{aligned}
$$

Proof. When $k=1$, the formula of Theorem 4.2 becomes completely trivial:

$$
p_{d+1, d}=(d+1) ! \cdot\left|\frac{1}{(d+1) !}\right|=1 .
$$

In the case $k=2$, we have a single sum where a $2 \times 2$ determinant appears:

$$
\begin{aligned}
p_{d+2, d} & =\sum_{\substack{a_{1}, a_{2} \geq 2 \\
a_{1}+a_{2}=d+2}}(d+2) ! \cdot\left|\begin{array}{cc}
\frac{1}{a_{1} !} & \frac{1}{(d+1) !} \\
1 & \frac{1}{a_{2} !}
\end{array}\right|=\sum_{a=2}^{d}\left(\left(\begin{array}{c}
d+2 \\
a
\end{array}\right)-(d+2)\right) \\
& =2^{d+2}-2(d+3)-(d-1)(d+2)=2^{d+2}-2-(d+1)(d+2) .
\end{aligned}
$$

If $k=d$, the formula of Theorem 4.2 gives an evaluation of Catalan numbers $\left(C_{n}\right)_{n \in \mathbf{N}}$. The fact that $p_{2 d, d}$ is the $d$-th Catalan number is clear from Proposition 3.1, since $d$-minimal permutations are in bijection with Young tableaux of rectangular shape having $d$ rows and 2 columns (see [St2]). Another combinatorial proof of this fact is given in $\mathrm{BP}$. Thus we get the following expression for Catalan numbers, which we have not been able to find in the literature:

$$
C_{d}=p_{2 d, d}=(2 d) ! \cdot\left|\begin{array}{ccccccc}
\frac{1}{2 !} & \frac{1}{3 !} & \frac{1}{4 !} & \frac{1}{5 !} & \frac{1}{6 !} & \cdots & \frac{1}{(d+1) !} \\
1 & \frac{1}{2 !} & \frac{1}{3 !} & \frac{1}{4 !} & \frac{1}{5 !} & \cdots & \frac{1}{d !} \\
1 & 1 & \frac{1}{2 !} & \frac{1}{3 !} & \frac{1}{4 !} & \cdots & \frac{1}{(d-1) !} \\
0 & 1 & 1 & \frac{1}{2 !} & \frac{1}{3 !} & \cdots & \frac{1}{(d-2) !} \\
0 & 0 & 1 & 1 & \frac{1}{2 !} & \cdots & \frac{1}{(d-3) !} \\
\vdots & \vdots & \vdots & \vdots & \vdots & \ddots & \vdots \\
0 & 0 & 0 & 0 & 0 & \cdots & \frac{1}{2 !}
\end{array}\right| .
$$

We close the section with the evaluation of $p_{d+3, d}$. As we stated above, to compute this value we have made extensive use of Maple. 
Theorem 4.4 The following equality holds:

$$
p_{d+3, d}=3^{d+3}-\left(d^{2}+4 d+7\right) \cdot 2^{d+2}+\frac{1}{2} d^{4}+\frac{5}{2} d^{3}+\frac{33}{4} d^{2}+6 d-8 .
$$

Proof. We just have to apply Theorem 4.2 in the case $k=3$, thus obtaining:

$$
p_{d+3, d}=\sum_{\substack{a, b, c \geq 2 \\
a+b+c=d+3}}(d+3) ! \cdot\left|\begin{array}{ccc}
\frac{1}{a !} & \frac{1}{(a+b-1) !} & \frac{1}{(d+1) !} \\
1 & \frac{1}{b !} & \frac{1}{(b+c-1) !} \\
\chi_{b=2} & 1 & \frac{1}{c !}
\end{array}\right| .
$$

The presence of the characteristic function $\chi_{b=2}$ suggests to consider two distinct cases.

i) In Formula (2), the partial sum for the tuples $(a, b, c)$ such that $b=2$ is:

$$
\begin{aligned}
& \sum_{\substack{a, c \geq 2 \\
a+c=d+1}}(d+3) ! \cdot\left|\begin{array}{ccc}
\frac{1}{a !} & \frac{1}{(a+1) !} & \frac{1}{(d+1) !} \\
1 & \frac{1}{2} & \frac{1}{(c+1) !} \\
1 & 1 & \frac{1}{c !}
\end{array}\right| \\
= & \sum_{a=2}^{d-1}(d+3) ! \cdot\left|\begin{array}{ccc}
\frac{1}{a !} & \frac{1}{(a+1) !} & \frac{1}{(d+1) !} \\
1 & \frac{1}{2} & \frac{1}{(d+2-a) !} \\
1 & 1 & \frac{1}{(d+1-a) !}
\end{array}\right| \\
= & \sum_{a=2}^{d-1}\left(\left(\begin{array}{c}
d+3 \\
a, 2, d+1-a
\end{array}\right)+\left(\begin{array}{c}
d+3 \\
a+1
\end{array}\right)+(d+2)(d+3)+\right. \\
& \left.-\frac{1}{2}(d+2)(d+3)-(d+3)\left(\begin{array}{c}
d+2 \\
a
\end{array}\right)-(d+3)\left(\begin{array}{c}
d+2 \\
a+1
\end{array}\right)\right) \\
= & \sum_{a=2}^{d-1}\left(\frac{(d+2)(d+3)}{2}\left(\begin{array}{c}
d+1 \\
a
\end{array}\right)+\frac{1}{2}(d+2)(d+3)-(d+2)\left(\begin{array}{c}
d+3 \\
a+1
\end{array}\right)\right) \\
= & \left(\begin{array}{c}
d+3 \\
2
\end{array}\right)\left(2^{d+1}-d-6\right)-(d+2)\left(2^{d+3}-d^{2}-7 d-14\right) .
\end{aligned}
$$

ii) The partial sum for the tuples $(a, b, c)$ such that $b \neq 2$ is a bit more 
complicated to compute but gives the following:

$$
\begin{aligned}
& \sum_{\substack{a, c \geq 2, b>2 \\
a+b+c=d+3}}(d+3) ! \cdot\left|\begin{array}{ccc}
\frac{1}{a !} & \frac{1}{(a+b-1) !} & \frac{1}{(d+1) !} \\
1 & \frac{1}{b !} & \frac{1}{(b+c-1) !} \\
0 & 1 & \frac{1}{c !}
\end{array}\right| \\
= & \sum_{\substack{a, c \geq 2, b>2 \\
a+b+c=d+3}}\left(\left(\begin{array}{c}
d+3 \\
a, b, c
\end{array}\right)+(d+2)(d+3)-(d+3)\left(\begin{array}{c}
d+2 \\
a
\end{array}\right)-(d+3)\left(\begin{array}{c}
d+2 \\
c
\end{array}\right)\right) \\
= & \sum_{a=2}^{d-2} \sum_{c=2}^{d-a}\left(\begin{array}{c}
d+3 \\
a, d+3-a-c, c
\end{array}\right)+\sum_{a=2}^{d-2} \sum_{c=2}^{d-a}(d+2)(d+3) \\
= & -\sum_{a=2}^{d-2} \sum_{c=2}^{d-a}(d+3)\left(\begin{array}{c}
d+2 \\
a
\end{array}\right)-\sum_{a=2}^{d-2} \sum_{c=2}^{d-a}(d+3)\left(\begin{array}{c}
d+2 \\
c
\end{array}\right) \\
= & \alpha+\beta-2 \gamma,
\end{aligned}
$$

if we set

$$
\begin{gathered}
\alpha=\sum_{a=2}^{d-2} \sum_{c=2}^{d-a}\left(\begin{array}{c}
d+3 \\
a, d+3-a-c, c
\end{array}\right), \quad \beta=\sum_{a=2}^{d-2} \sum_{c=2}^{d-a}(d+2)(d+3) \\
\text { and } \gamma=\sum_{a=2}^{d-2} \sum_{c=2}^{d-a}(d+3)\left(\begin{array}{c}
d+2 \\
a
\end{array}\right) .
\end{gathered}
$$

We compute the two terms $\alpha$ and $\gamma$ using Maple:

$$
\begin{aligned}
& \alpha=3^{d+3}-(d+11)(d+6) \cdot 2^{d}+\left(d^{3}+10 d^{2}+37 d+51\right) \\
& \gamma=\left(d^{2}-d-12\right) \cdot 2^{d+1}-\left(\frac{1}{2} d^{3}-d^{2}-\frac{41}{2} d-39\right) .
\end{aligned}
$$

Instead, the term $\beta$ is of course very easy to compute directly:

$$
\beta=(d+2)(d+3) \frac{(d-3)(d-2)}{2}=\frac{\left(d^{2}-4\right)\left(d^{2}-9\right)}{2} .
$$

Thus using Formula (4) we get:

$$
\begin{aligned}
& \sum_{\substack{a, c \geq 2, b>2 \\
a+b+c=d+3}}(d+3) ! \cdot\left|\begin{array}{ccc}
\frac{1}{a !} & \frac{1}{(a+b-1) !} & \frac{1}{(d+1) !} \\
1 & \frac{1}{b !} & \frac{1}{(b+c-1) !} \\
0 & 1 & \frac{1}{c !}
\end{array}\right| \\
= & 3^{d+3}-\left(5 d^{2}+13 d+18\right) \cdot 2^{d}+\left(\frac{1}{2} d^{4}+2 d^{3}+\frac{3}{2} d^{2}-4 d-9\right)(5)
\end{aligned}
$$


Now, to finish the proof of our theorem, we just have to sum the result of (3) and (5), thus obtaining:

$$
p_{d+3, d}=3^{d+3}-\left(d^{2}+4 d+7\right) \cdot 2^{d+2}+\frac{1}{2} d^{4}+\frac{5}{2} d^{3}+5 d^{2}+6 d+1 .
$$

Table 11 shows the first few terms of the sequence $\left(p_{d+3,3}\right)_{d}$.

\begin{tabular}{|r|c|c|c|c|c|c|c|}
\hline$d$ & 3 & 4 & 5 & 6 & 7 & 8 & 9 \\
\hline$p_{d+3,3}$ & 5 & 84 & 686 & 3936 & 18387 & 75372 & 283052 \\
\hline
\end{tabular}

Table 1: The first few terms of the sequence $\left(p_{d+3,3}\right)_{d}$

\section{$5 \quad$ A generalization}

The main motivation of the present paper is the study of $d$-minimal permutations and, in particular, their enumeration. Our approach is based on a bijection between the set of $d$-minimal permutations and a special class of skew Young tableaux, namely those in which every pair of consecutive rows has precisely two columns in common. Denote by $\operatorname{SkYT}_{2}(n, k)$ this set of tableaux, $n$ being the number of cells and $k$ the number of rows. We can generalize this setting in a very natural way, by defining the set $S k Y T_{h}(n, k)$ of skew Young tableaux having $n$ cells and $k$ rows such that any two consecutive rows have precisely $h \geq 1$ columns in common. In this final section we wish to relate these tableaux with some families of permutations, as well as to describe some enumerative results for low values of $h$.

Our first result is a generalization of Theorem 3.1.

Theorem 5.1 The set $S k Y T_{h}(d+k, k)$ is in bijection with the set of permutations of length $d+k$ having exactly $d$ descents and satisfying the following property (call it $D E S_{h}$ ):

$D E S_{h}\left\{\begin{array}{l}\text { for every } i \leq h-1, \text { if one deletes } i \text { elements of a permutation } \\ \text { and renames the remaining elements in the usual way, } \\ \text { the resulting permutation has precisely } d-i \text { descents. }\end{array}\right.$

Proof. Denote with $S_{d+k}^{(h)}(d)$ the set of permutations of length $d+k$ having precisely $d$ descents and satisfying $D E S_{h}$. Define a map $f: S_{d+k}^{(h)}(d) \rightarrow$ $S k Y T_{h}(d+k, k)$ by suitably generalizing the one given in Proposition 3.1: starting from the bottom, the $i$-th row of the tableau consists of the elements 
of the $i$-th descending run of $\pi$, and two consecutive rows are required to have exactly $h$ columns in common. We claim that this map is well-defined. Indeed suppose, ab absurdo, that in $f(\pi)$ there is a column in which $a$ is above $b$ and $a<b$. Without loss of generality, we can assume that $a$ is in the cell immediately above $b$. Then, removing the $h-1$ entries of $\pi$ preceding $a$ and following $b$ and belonging to the columns common to the rows of $a$ and $b$, we obtain a permutation not satisfying $D E S_{h}$ (the number of descents is easily seen to be $d-h+2$ ), as shown on Figure 5. The fact that $f$ is injective and surjective is trivial, and follows directly from its definition.

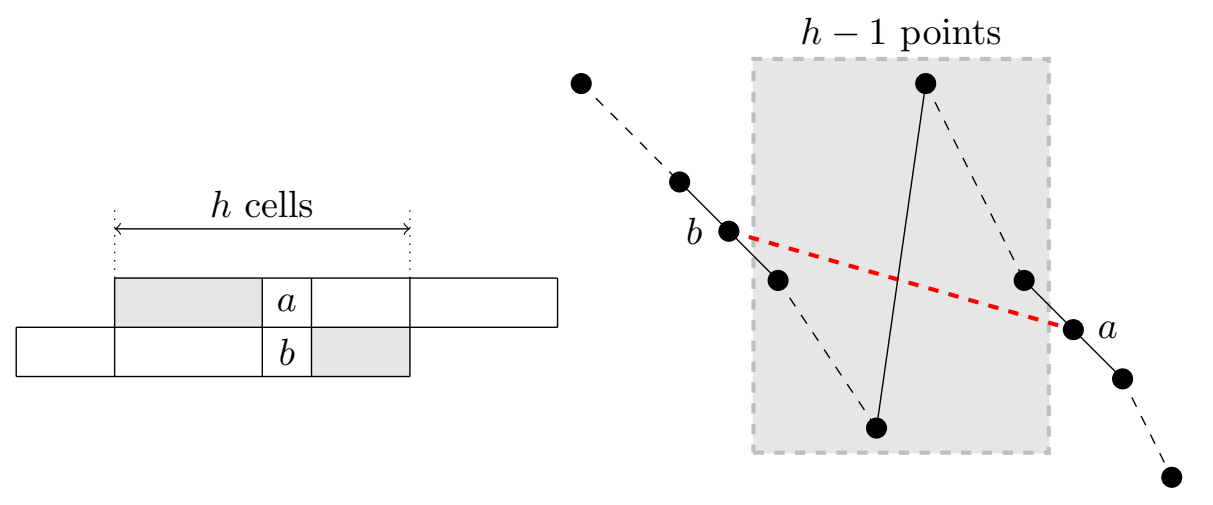

Figure 5: Proof of Theorem 5.1. The gray area corresponds to the $h-1$ points removed in the proof.

It is clear that, when $h=2$, we get precisely Theorem 3.1, since the resulting class of permutations is that of $d$-minimal permutations.

We also have a characterization of the above classes of permutations in terms of patterns, which follows quite easily from the above theorem, and so will be stated without proof.

Theorem 5.2 A permutation $\sigma$ belongs to $S_{d+k}^{(h)}(d)$, for some $k$, if and only if it has exactly d descents and its ascents occur in the middle of a consecutive pattern of the form $\pi=\pi_{1} \pi_{2}$, where $\pi_{1}$ and $\pi_{2}$ are words of the same length $h$, both decreasing and $\pi_{1}<\pi_{2}$ componentwise.

Having introduced this generalized setting, it is natural to ask what happens when $h<2$.

If $h=1$, what we obtain is the class of permutations having exactly $d$ descents. It is well known that the number of permutations of length $n$ having $d$ descents is given by the Eulerian number $E_{n, d}$ (sequence A008292 in [S1]). Thanks to our approach, we find a determinant expression of Eulerian numbers which is believed to be new. Once again, the key ingredient to obtain such a formula is of course Theorem 4.1. 
Theorem 5.3 The number $E_{d+k, d}$ of permutations of length $d+k$ having exactly d descents (i.e. satisfying condition $D E S_{1}$ ) is

$$
E_{d+k, d}=\sum_{\substack{a_{1}, a_{2}, \ldots, a_{k} \geq 1 \\ a_{1}+a_{2}+\cdots+a_{k}=d+k}}(d+k) ! \cdot \operatorname{det}\left(B\left(a_{1}, \ldots, a_{k}\right)\right),
$$

where $B\left(a_{1}, \ldots, a_{k}\right)$ is the following matrix:

$$
\left(\begin{array}{cccccc}
\frac{1}{a_{1} !} & \frac{1}{\left(a_{1}+a_{2}\right) !} & \frac{1}{\left(a_{1}+a_{2}+a_{3}\right) !} & \cdots & \frac{1}{\left(a_{1}+\cdots+a_{k-1}\right) !} & \frac{1}{\left(a_{1}+\cdots+a_{k}\right) !} \\
1 & \frac{1}{a_{2} !} & \frac{1}{\left(a_{2}+a_{3}\right) !} & \cdots & \frac{1}{\left(a_{2}+\cdots+a_{k-1}\right) !} & \frac{1}{\left(a_{2}+\cdots+a_{k}\right) !} \\
0 & 1 & \frac{1}{a_{3} !} & \cdots & \frac{1}{\left(a_{3}+\cdots+a_{k-1}\right) !} & \frac{1}{\left(a_{3}+\cdots+a_{k}\right) !} \\
\vdots & \vdots & 1 & \ddots & \vdots & \vdots \\
0 & 0 & \cdots & \ddots & \frac{1}{a_{k-1} !} & \frac{1}{\left(a_{k-1}+a_{k}\right) !} \\
0 & 0 & 0 & \cdots & 1 & \frac{1}{a_{k} !}
\end{array}\right) .
$$

In other words, $B\left(a_{1}, \ldots, a_{k}\right)$ is the $k \times k$ matrix whose entries $b_{i, j}$ obey the following equalities:

$$
\begin{aligned}
a_{i, j} & =\frac{1}{\left(a_{i}+\cdots+a_{j}\right) !}, \quad \text { when } i \leq j, \\
a_{i, i-1} & =1, \\
a_{i, j} & =0, \quad \text { when } i \geq j+2 .
\end{aligned}
$$

Proof. The proof essentially follows the same lines of the proof of Theorem 4.2; just observe that, in this case, it is $\lambda_{i+1}-\mu_{i}=1$.

Moreover, the determinant of the matrix $B\left(a_{1}, \ldots, a_{k}\right)$ has a very neat recursive expression, from which a closed formula can be deduced.

Proposition 5.1 Set $D\left(a_{1}, \ldots, a_{k}\right)=\operatorname{det}\left(B\left(a_{1}, \ldots, a_{k}\right)\right)$. Then

$$
D\left(a_{1}, a_{2}, \ldots, a_{k}\right)=\frac{1}{a_{1} !} \cdot D\left(a_{2}, a_{3}, \ldots, a_{k}\right)-D\left(a_{1}+a_{2}, a_{3}, \ldots, a_{k}\right) .
$$

Proof. Just expand $D\left(a_{1}, \ldots, a_{k}\right)$ with respect to its first column.

Corollary 5.1 The following formula holds:

$$
D\left(a_{1}, \ldots, a_{k}\right)=\sum_{i=1}^{k}(-1)^{k-i} \cdot \sum_{\substack{\alpha=\left(\alpha_{1}, \ldots, \alpha_{i}\right) \\ \alpha \in \mathbf{P L}\left(a_{1}, \cdots, a_{k}\right)}} \frac{1}{\left|\alpha_{1}\right| ! \cdot \ldots \cdot\left|\alpha_{i}\right| !},
$$

where $\mathbf{P L}\left(a_{1}, \cdots, a_{k}\right)$ denotes the set of linear partitions of the totally ordered set $\left\{a_{1}, \ldots, a_{k}\right\}$ and $\left|\alpha_{i}\right|$ is the sum of the elements of the block $\alpha_{i}$. 
Proof. We start by observing that, when $k=1$, the outer sum of the r.h.s of (6) reduces to a single summand (for $i=1$ ), as well as the inner sum, which has the unique summand $\frac{1}{a_{1} !}$. Moreover, when $k=2$, the r.h.s. of (6) consists of two summands, which are $(-1) \cdot \frac{1}{\left(a_{1}+a_{2}\right) !}($ for $i=1)$ and $\frac{1}{a_{1} !} \cdot \frac{1}{a_{2} !}($ for $i=2)$, and this coincides with the expression of $D\left(a_{1}, a_{2}\right)$.

We can now conclude our proof using an inductive argument. The set $\mathbf{P L}\left(a_{1}, \cdots, a_{k}\right)$ can be partitioned into two subsets, namely the linear partitions in which $a_{1}$ occurs as a singleton (call this subset $X$ ) and the linear partitions in which $a_{1}$ occurs in a block of cardinality at least 2 (call this subset $Y)$. Using this partition of $\mathbf{P L}\left(a_{1}, \cdots, a_{k}\right)$ we can split the sum in the r.h.s of (6) into two sums, the first taking into account the contribution of $X$ and the second taking into account the contribution of $Y$. We thus obtain the following equalities:

$$
\begin{aligned}
\sum_{\substack{\alpha=\left(\alpha_{1}, \ldots, \alpha_{i}\right) \\
\alpha \in \mathbf{P L}\left(a_{1}, \cdots, a_{k}\right)}} \frac{1}{\left|\alpha_{1}\right| ! \cdot \ldots \cdot\left|\alpha_{i}\right| !} & =\frac{1}{a_{1} !} \cdot \sum_{\substack{\beta=\left(\beta_{1}, \ldots, \beta_{j}\right) \\
\beta \in \mathbf{P L}\left(a_{2}, \cdots, a_{k}\right)}} \frac{1}{\left|\beta_{1}\right| ! \cdot \ldots \cdot\left|\beta_{j}\right| !} \\
& +\sum_{\substack{\gamma=\left(\gamma_{1}, \ldots, \gamma_{t}\right) \\
\gamma \in \mathbf{P L}\left(\left\{a_{1}, a_{2}\right\}, a_{3}, \cdots, a_{k}\right)}} \frac{1}{\left|\gamma_{1}\right| ! \cdot \ldots \cdot\left|\gamma_{t}\right| !},
\end{aligned}
$$

whence, using the induction hypothesis and the above proposition:

$$
\begin{aligned}
& \sum_{i=1}^{k}(-1)^{k-i} \cdot \sum_{\substack{\alpha=\left(\alpha_{1}, \ldots, \alpha_{i}\right) \\
\alpha \in \mathbf{P L}\left(a_{1}, \cdots, a_{k}\right)}} \frac{1}{\left|\alpha_{1}\right| ! \cdot \ldots \cdot\left|\alpha_{i}\right| !} \\
& =\frac{1}{a_{1} !} \cdot D\left(a_{2}, \ldots, a_{k}\right)-D\left(a_{1}+a_{2}, a_{3}, \ldots, a_{k}\right)=D\left(a_{1}, \ldots, a_{k}\right),
\end{aligned}
$$

as desired.

Remark. An alternative approach to the case $h=1$ could be done via the notion of Hessenberg matrix. An (upper) Hessenberg matrix is a square matrix having zero entries below the first subdiagonal. Hessenberg matrices prove their usefulness especially in numerical analysis and computer programming, being a sort of normal form to which any square matrix can be reduced in a finite number of steps. There are also some papers in the literature concerning the evaluation of the determinant of certain Hessenberg matrices having special form (see for instance [BS] and [LCT]). In [T], the determinant of Hessenberg matrices having all the elements of the first subdiagonal equal to 1 is considered (this is precisely the kind of matrices we meet in Theorem 5.3).

Theorem 5.1 does not have meaning when $h=0$. The corresponding set $S k Y T_{0}(n, k)$ consists of all skew Young tableaux having $n$ cells and $k$ rows 
such that any two consecutive rows only have the corners of two cells in common. In this case, it is immediate to see that $S k Y T_{0}(n, k)$ is in bijection with all surjective functions from an $n$-set to a $k$-set: just interpret the elements of a tableau as balls and the rows of a tableau as boxes. Thus we get immediately that $\left|S k Y T_{0}(n, k)\right|=k ! \cdot S(n, k)$, where the $S(n, k)$ 's are the Stirling numbers of the second kind. We can also use Theorem 4.1 to get an analog of Theorems 4.2 and 5.3; indeed, we can derive the following formula:

$$
\left|S k Y T_{0}(d+k, k)\right|=\sum_{\substack{a_{1}, a_{2}, \ldots, a_{k} \geq 1 \\ a_{1}+a_{2}+\cdots+a_{k}=d+k}}(d+k) ! \cdot \operatorname{det}\left(C\left(a_{1}, \ldots, a_{k}\right)\right),
$$

where $C\left(a_{1}, \ldots, a_{k}\right)$ is the following triangular matrix:

$\left(\begin{array}{cc}\frac{1}{a_{1} !} & \frac{1}{\left(a_{1}+a_{2}+1\right) !} \\ 0 & \frac{1}{a_{2} !} \\ 0 & 0 \\ \vdots & \vdots \\ 0 & 0 \\ 0 & 0\end{array}\right.$

$\begin{array}{cc}\frac{1}{\left(a_{1}+a_{2}+a_{3}+2\right) !} & \cdots \\ \frac{1}{\left(a_{2}+a_{3}+1\right) !} & \cdots \\ \frac{1}{a_{3} !} & \cdots \\ \ddots & \\ \cdots & \\ 0 & \cdots\end{array}$
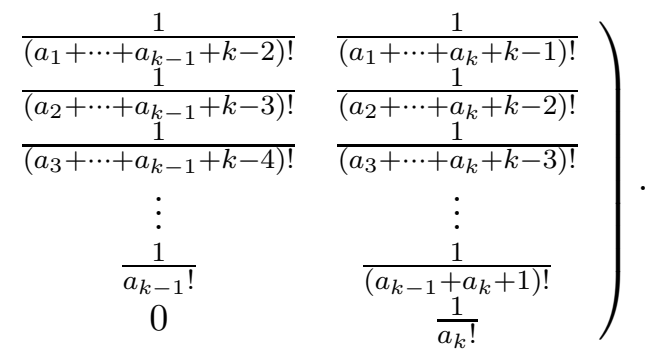

From here it is then immediate to obtain $\left|S k Y T_{0}(d+k, k)\right|=$ $\sum_{\substack{a_{1}, a_{2}, \ldots, a_{k} \geq 1 \\ a_{1}+a_{2}+\cdots+a_{k}=d+k}}\left(\begin{array}{c}d+k \\ a_{1}, \ldots a_{k}\end{array}\right)$ which is known to be the number of surjective functions from an $(d+k)$-set to a $k$-set, as already shown a few lines above.

\section{Further work}

Even if our approach to the enumeration of $d$-minimal permutations allows us to completely solve the problem from a purely theoretical point of view, it is doubtless that its application to concrete cases shows some technical difficulties. This is of course due to the intrinsic complexity of the sums of determinants appearing in Theorem 4.2. However, it seems plausible that at least a few more cases than those we deal with in the present paper can be managed by means of our technique.

Another interesting problem that remains untouched concerns the study of the structure of the poset determined by a minimal permutations with $d$ descents, defined in [BP] and recalled in Section 2 here. For instance, one can observe that a $d$-minimal permutation corresponds to a linear extension of the associated poset. Moreover, an interesting (and classical) line of research could be the investigation of the properties of the distributive lattice of the sup-irreducibles of these posets. 


\section{References}

[A] A. C. Aitken, The monomial expansion of determinantal symmetric functions Proc. Royal Soc. Edinburgh (A) 61 (1943) 300-310.

[BaRo] Y. Baryshnikov, D. Romik, Enumeration formulas for Young tableaux in a diagonal strip Israel J. Math. 178 (2010) 157-186.

[BS] A. T. Benjamin, M. A. Shattuck, Recounting determinants for a class of Hessenberg matrices Integers 7 (2007) \# A55 (7 pp.).

[BP] M. Bouvel, E. Pergola, Posets and permutations in the duplicationloss model: minimal permutations with d descents Theoret. Comput. Sci. 411 (2010) 2487-2501.

[BoRo] M. Bouvel, D. Rossin, A variant of the tandem duplication - random loss model of genome rearrangement Theoret. Comput. Sci. 410 (2009) 847-858.

[LCT] H.-C. Li, Y.-M. Chen, E.-T. Tan, Counting determinants of Fibonacci-Hessenberg matrices using LU factorizations Integers 9 (2009) \#A37 (23 pp.).

[MY] T. Mansour, S. H. F. Yan, Minimal permutations with d descents European J. Combin. 31 (2010) 1445-1460.

[P] V. R. Pratt, Computing permutations with double-ended queues, parallel stacks and parallel queues Proceedings of the fifth annual ACM symposium on Theory of Computing, 1973, 268-277.

[Sl] N. J. A. Sloane, The On-Line Encyclopedia of Integer Sequences at http://www.research.att.com/ njas/sequences/index.html.

[St1] R. P. Stanley, On the enumeration of skew Young tableaux Adv. Appl. Math. 30 (2003) 283-294.

[St2] R. P. Stanley, Enumerative Combinatorics, Vol. 2 Cambridge University Press, Cambridge, 1999.

[T] U. Tamm, The determinant of a Hessenberg matrix and some applications in discrete mathematics preprint. 